\title{
Radiobiological effects of the interruption time in radiation therapy with photon beams based on the microdosimetric kinetic model
}

\section{CURRENT STATUS: UNDER REVIEW}

Radiation Oncology $\triangle B M C$

Hisashi Nakano

Osaka University

hisankn@gmail.comCorresponding Author

ORCiD: https://orcid.org/0000-0002-9023-880X

Daisuke Kawahara

Hiroshima University

Satoshi Tanabe

Nihon Shika Daigaku Niigata Seimei Shigakubu Daigakuin Niigata Seimei Shigakubu Kenkyuka

Satoru Utsunomiya

Niigata University

Takeshi Takizawa

Niigata University

Hidefumi Aoyama

Hokkaido University

DOI:

10.21203/rs.3.rs-17887/v1

\section{SUBJECT AREAS}

Oncology

\section{KEYWORDS}

External photon beams, Sublethal damage repair, Interruption time, Monte Carlo simulation, Microdosimetric kinetic model 
Abstract

Background: We investigated the effect of the interruption time on the radiobiological effectiveness with photon beams based on a modified microdosimetric kinetic model.

Background: The interruption time is used is that irradiation interruption occurs at sites and operations such as the gantry, collimator, couch rotation, and patient set-up in radiotherapy. However, the effect of interruption time on photon beams for tumor cells is little evaluated.

Methods: The dose-mean lineal energy $\mathrm{y}_{\mathrm{D}}(\mathrm{keV} / \mu \mathrm{m})$ of $6 \mathrm{MV}$ photon beams was calculated by the particle and heavy ion transport system (PHITS). We set the absorbed dose to 2 or 8 Gy, and the interruption time $(\tau)$ was set to $1,3,5$, or $10 \mathrm{~min}$. The biological parameters values were acquired from a human non-small cell lung cancer cell line ( $\mathrm{NCl}-\mathrm{H} 460)$ for the mMK model. We used two-field and four-field irradiation with a constant dose rate ( $3 \mathrm{~Gy} / \mathrm{min}$ ); the photon beams were paused for interruption time $\tau$. We calculated the relative biological effectiveness (RBE) to evaluate the interruption time's effect compared with photon beams that were not interrupted as a reference.

Results: The RBE with four-field irradiation for 8 Gy was decreased to $0.997,0.992,0.987$, and 0.975 for $\tau=1,3,5$, and 10 min, respectively. The decrease in the RBE was within 3\% compared with the non-interrupted photon beams.

Conclusions: The effect of RBE was decreased by increasing the interruption time, indicating that an escalation of the prescribed dose might be necessary when the interruption time was large. Full-text

Due to technical limitations, full-text HTML conversion of this manuscript could not be completed. However, the manuscript can be downloaded and accessed as a PDF.

Figures 


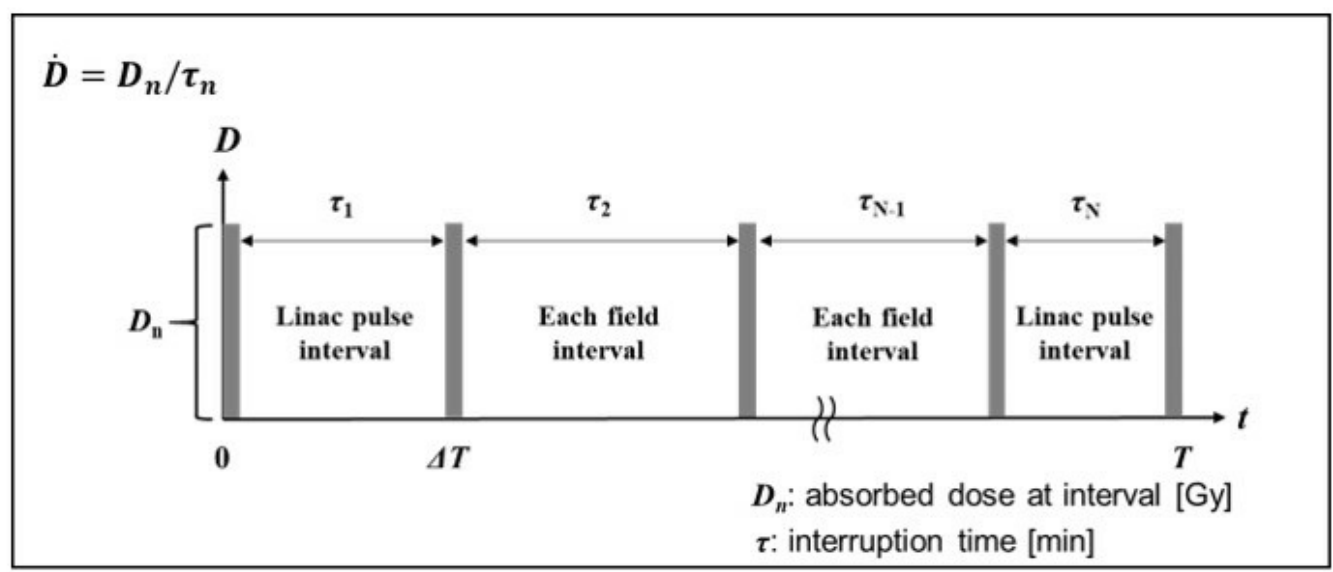

Figure 1

Diagram of the mMK model, taking into consideration the interruption time of photon beams. 


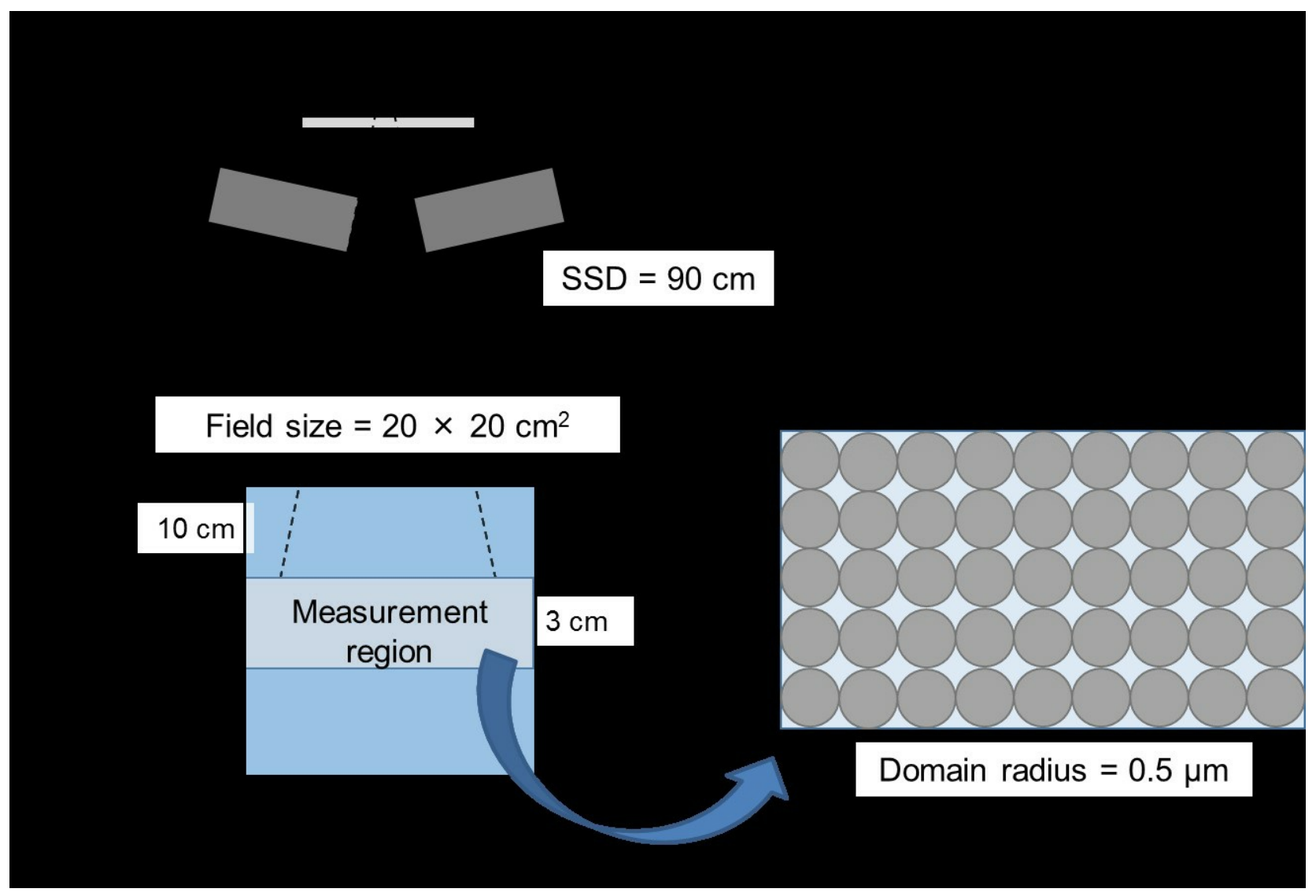

Figure 2

Irradiation geometry for the Monte Carlo calculations with $6 \mathrm{MV}$ photon beams. The radius of the domain was set to $0.5 \mu \mathrm{m}$ in the $3-\mathrm{cm}$-wide measurement region with the waterequivalent phantom. 
(a)

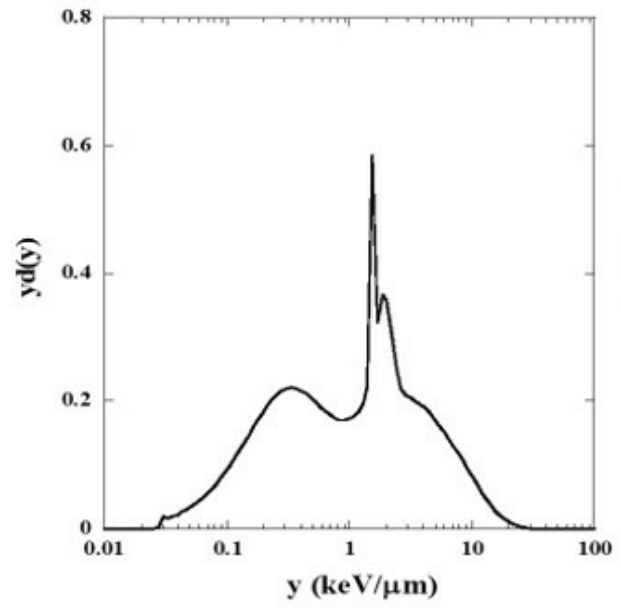

(b)

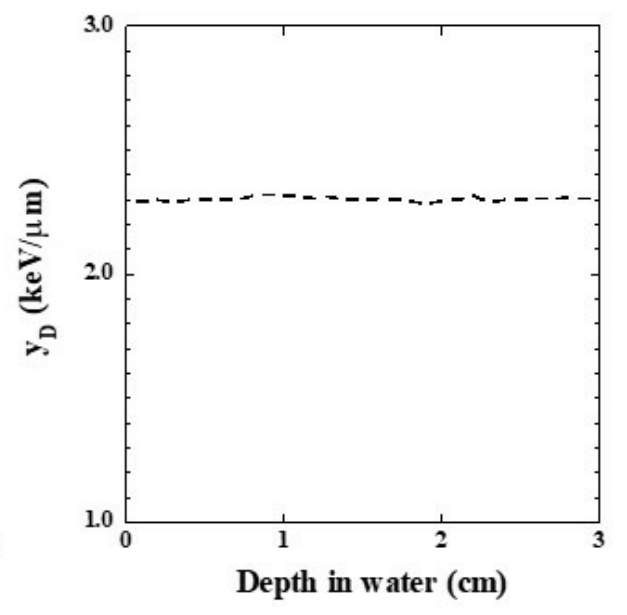

Figure 3

The microdosimetric energy distributions $y D$ as a function of $y$-yd(y) for 6 MV photon beams at a depth of $10 \mathrm{~cm}$ in a water-equivalent phantom (a). The relationship between the depth (which was $3 \mathrm{~cm}$ from the measurement point in the water equivalent phantom) and the dose-mean lineal energy yD for the $6 \mathrm{MV}$ photon beams (b). 
(a)

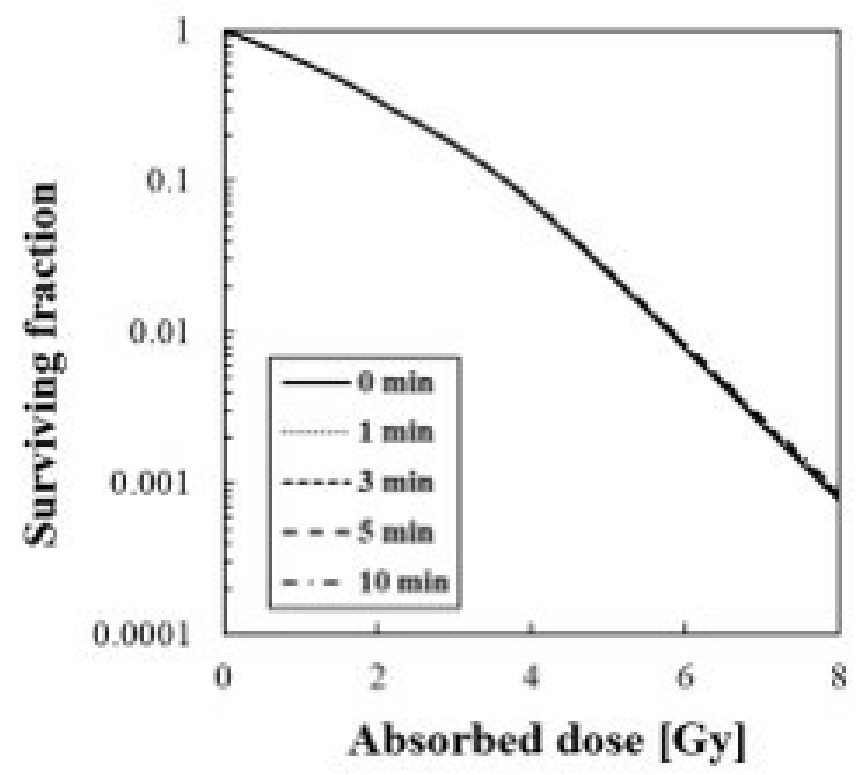

(b)

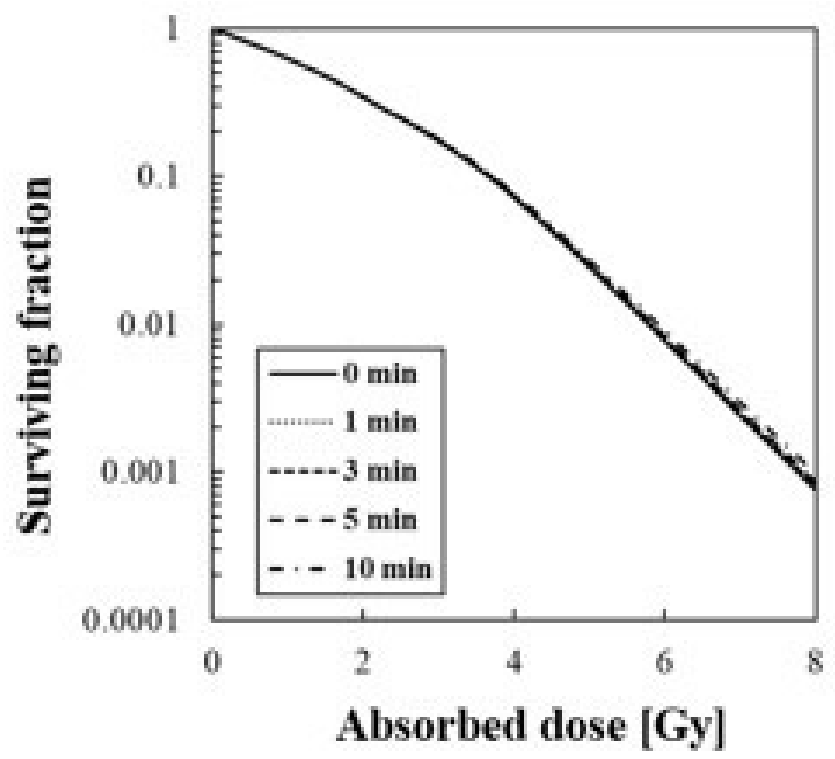

Fig. 4.

Figure 4

The effect of the interruption time on the SF for 6 MV photon beams with two-field (a) and four-field (b) irradiation for the various interruption times. 
(a)

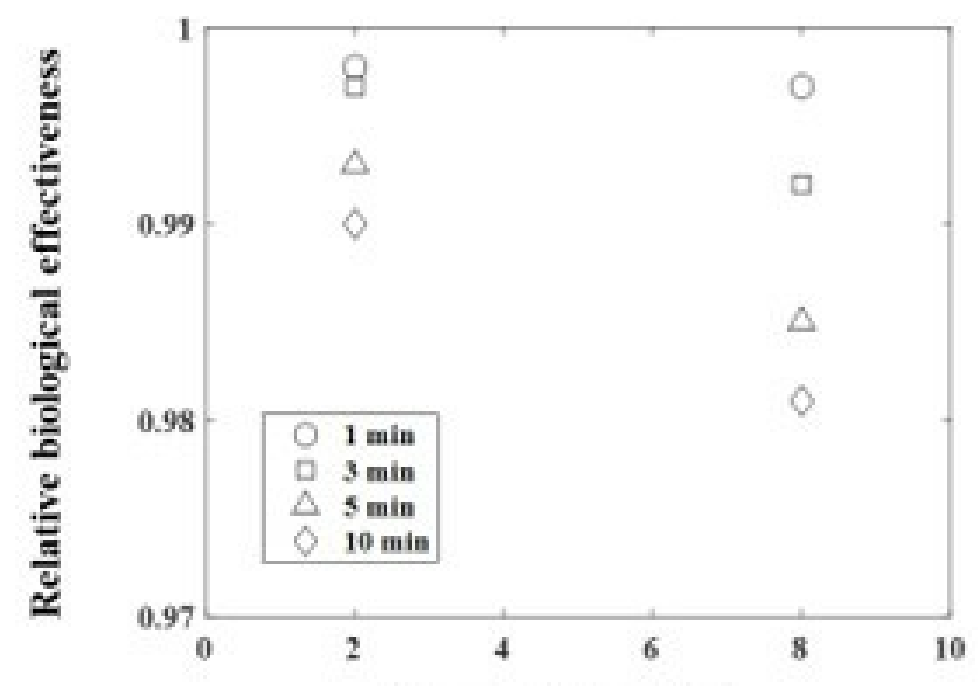

Absorbed dose $[\mathrm{Gy}]$

(b)

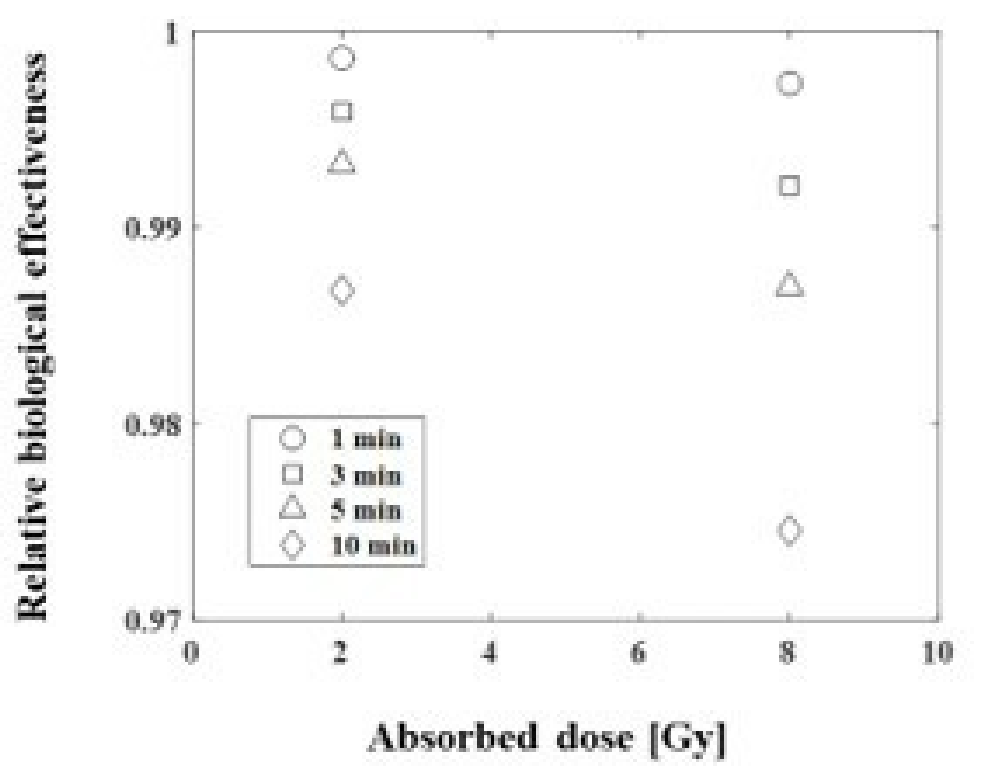

Figure 5

The effect of the interruption time on the RBE with two-field (a) and four-field (b) irradiation for the various interruption times. 


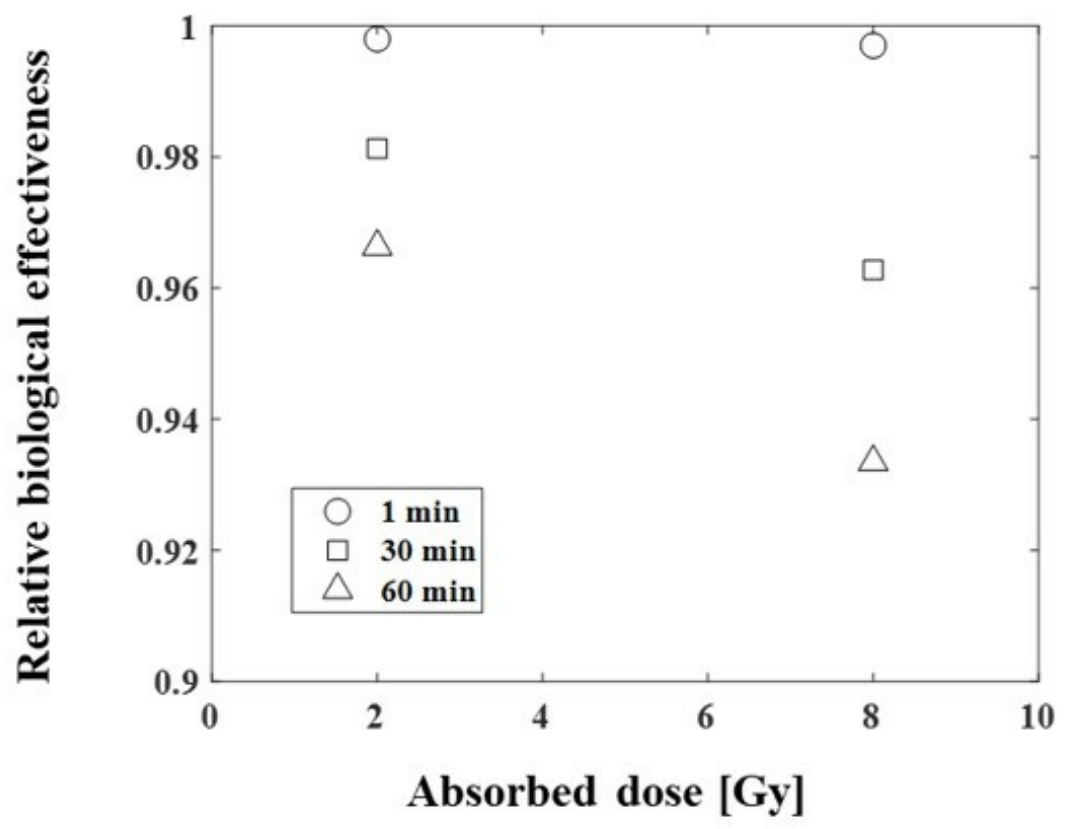

Figure 6

The effect of prolonging the interruption time ( 1 to $60 \mathrm{~min}$ ) on the RBE when two-field irradiation was used. 
(a)

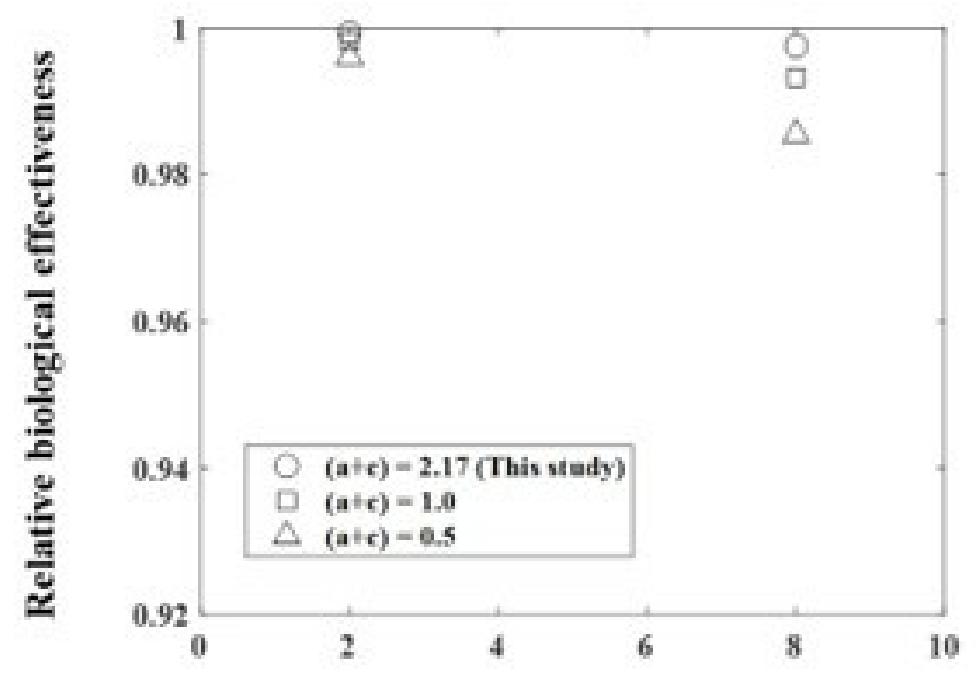

Absorbed dose [Gy]

(b)

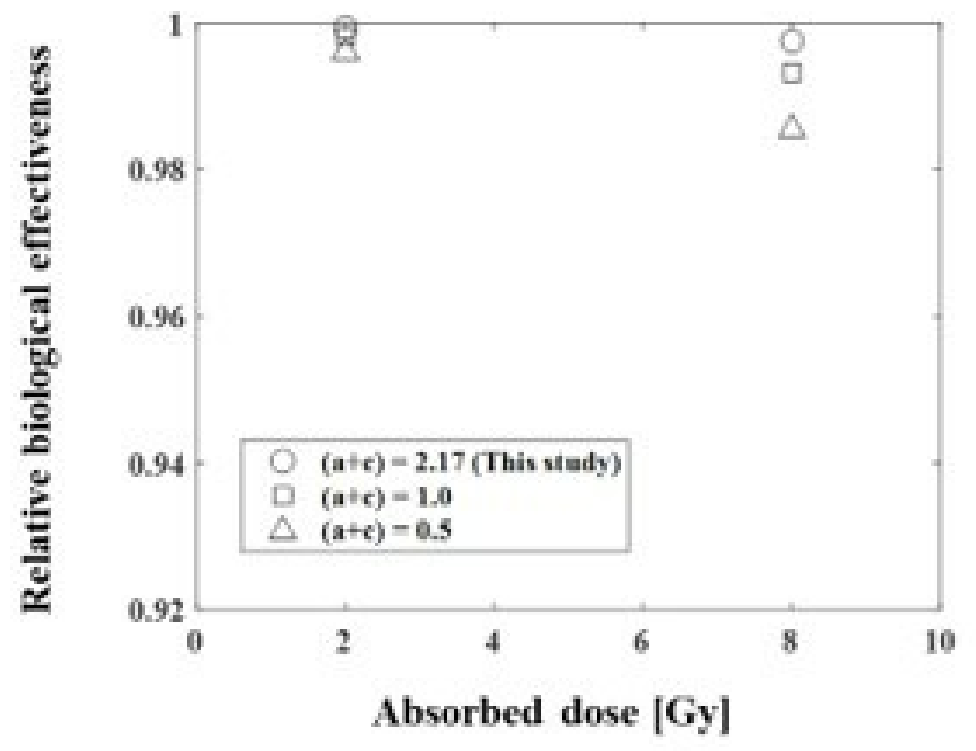

Fig. 7.

Figure 7

The effect of RBE as a function of the cell-specific repair rate $(a+c)$ with $\tau=1 \mathrm{~min}(a)$ and $\tau=10 \mathrm{~min}(\mathrm{~b})$ using two-field irradiation. 
